\title{
Significance of Context Sensitiveness in Content based Image Retrieval System and Bridging the Semantic Gap
}

\author{
N.Karthikeyan \\ Research Scholar, Bharathiar University, \\ Coaimbatore. Head (B.C.A Dept), \\ Department of Computer Applications, \\ Srimad Andavan Arts\&Science College, \\ Tiruchitrapalli, Tamil Nadu, India
}

\author{
R.Dhanapal Ph.D., FIASTED \\ MIACSIT, MIAENG \\ Professor \& Head, Research Department of \\ Computer Applications, \\ Easwari Engineering College \\ Chennai - 600089
}

\begin{abstract}
The increase in discovery of faster processors and high capacity storage devices has triggered the development of search beyond plain text. 2D searches have started becoming prevalent, that helps users in obtaining images according to their queries. In this paper, we discuss the principal challenges facing CBIR systems and ways in which they could be overcome. Discussion of various solutions provided for improvising the CBIR technique is discussed. The paper concludes with the directions for future research, along with implementation suggestions that will be helpful during CBIR implementations.
\end{abstract}

Keywords: CBIR, Semantic Gap, Context Sensitive Retrieval, Load Balancing, Parallelization Techniques

\section{INTRODUCTION}

An Image Retrieval system can be basically considered as a binary classifier that divides the image repository into two classes; the relevant class and the irrelevant class $[1,2]$. The relevance of these images are based on the image provided by the user, called the query. The query is an image and the user wishes to find images in the database that contain objects present in the query image. Hence the main functionality of the system is to initially ascertain all the objects contain in the image, and then find images that match all or some of these objects. The images retrieved may be quite different from the query image. But as long as they contain the objects of interest they are considered to be relevant. This is sometimes referred to as search for objects to distinguish it with methods that attempt to match whole images. However, not all the images in the repository contain labels for the objects contained in them, and not all the objects in the images are labeled.

The most important step is to understand the nature of the problem and how the image can be transformed into a mathematical representation without loss of information about what we consider to be the important aspects of an image. For the case of OCR the important elements were strokes and their spatial relationships (but not their color, or, within limits, their thickness). If color and texture are important (for example, to identify a lesion in a medical image) then we need texture and color descriptors that are accurate enough to represent uniquely the textures and colors of interest.

The remainder of this paper is structured as follows; section II provides

\section{PRINCIPAL CHALLENGES FACING CBIR}

The basic problems faced by CBIR systems include heterogeneity, where inputs of various formats are being used, incompleteness, where the user cannot expect the system to contain the exact matching for the user string, inconsistency and the large database size. This makes the discovery of useful patterns difficult, expensive, and perhaps even impossible to implement in some cases. The main reason seems to be that the lessons about feature selection and the "curse of dimensionality" in pattern recognition have been ignored in CBIR. Because there is little connection between pixel statistics and the human interpretation of an image (the "semantic gap") the use of large number of generic features makes highly likely that results will not be scalable. Even people with colour blindness don't feel the difference in real world until tested for it. So colour or luminance alone could not play a much important role.

There is very little support for exploratory search and scaling to very large collections is problematic. Moreover, the assumption that users are always able to formulate an appropriate query is questionable. The problem of estimating the relative significance of different features pertains to information retrieval in general. It is however greatly compounded in the case of image retrieval in two significant ways: First, while documents readily suggest a representation in terms of their constituent words, images do not generally admit to such a natural decomposition into semantic atoms. This renders image representations to some extent arbitrary. Secondly, images typically admit to a multitude of different meanings, each of which may have its own set of supporting visual features.

Users may, not have a well-defined information need in the first place and may simply wish to explore the image collection. Should a concrete information need exist, users are unlikely to have a query image at their disposal to express it. Moreover, nearest neighbor search requires efficient indexing structures that do not degrade to linear complexity with a large number of dimensions [3].

Many supervised learning approaches could be employed to solve this problem. Unfortunately, they are confronted by two main challenges. The first one is that the annotated or labeled training samples are too limited. Generally, the labels are provided by queries and relevance feedbacks, which will not be many. Limited training data would only result in weak classification .Another challenge is the dimensionality of learning, since high dimensional visual data would pose practical difficulties for feature weighting, selecting and 
dimensionality reduction. Limited training data would also prevent effective dimensionality reduction schemes.

The current CBIR techniques will not hold on collections of images other than the ones used during the development of the method. In other words, the transformation from images to features (or other descriptors) is many-to-one and when the data set is relatively small, there are no collisions. But as the size of the set increases unrelated images are likely to be mapped into the same features.

The way a Human Being looks at an image is completely different from that of a computerized program. It is context sensitive and not just content based. Our interpretation is semantics oriented and hence the need for a semantics based approach to bridge the gap. The semantic interpretation of an image has very little to do with statistics of the values of the pixels. Images tend to maintain their semantics even though their luminance or colour histograms are made flat.

A well-known observation from research in vision is that humans rely a lot on broad context and are likely to ignore local details. This suggests that reliance on low-level measurements may produce results different from those of a human viewer. The human visual system is able to ignore local cues once a high level interpretation has been achieved.

So it is not enough to look at parts of an image, we must also look at the overall composition. An operational reason for the difficulty is that the image data (values of pixels stored in bytes) are very far from the interpretation of images by human observers. This is not the case with text where the stored data are much closer to human interpretation. Text consists of words that are well-defined models of concepts so that communication amongst humans is possible. These symbols have a well-defined representation in terms of bits, so going from digital storage to a human understandable representation is a matter of table look up.

In text retrieval system users are free to compose queries using several words but this not the case with image retrieval where the user is usually asked to provide a query image or a sketch. Some researchers have recognized this and solutions include area selection in an image, semantic labels and the use of multiple images as well as feedback. All these approaches improve the performance of an image retrieval system.

However the challenge remains because the user must perform some image manipulation work, a more demanding task than typing words. A fairer comparison between image and text retrieval is to consider retrieval not of words but of concepts. It turns out that the state of the art of text retrieval based on concepts is not particularly advanced. Searching text by content is quite hard when the content we are looking for is a concept rather than a phrase. A computer search is effective only as long as we look for byte patterns. In the case of text, words are mapped easily into byte patterns, so searching for words is effective. This should give us a hint why contentbased image searching is hard.

We cannot have a mathematical representation without loss of information for the general category of "all images" but we have a good chance of achieving that by confining the problem into retrieving images from a particular category only (for example, matching an iris scan to a collection of iris scans).

\section{CBIR METHODS : A SURVEY}

Efficient categorization of images can be performed, if the mapping between semantics and image features is well defined by the system. By this process, the semantic gap can be reduced. The representation of features can be made without loss of information, in such a way that only images with similar interpretation and no others are mapped into the same set of features. Examples are found in the pattern recognition literature, including OCR and fingerprint recognition. If the context is known, we can deduce that the object of interest occupies a particular area in the image or is close some other well defined feature. The context can be taken advantage in the design and selection of the features. If the matching of images are performed with careful scrutiny, categorization might be easier. This is a process that humans are not very good at and it is likely that machines can match or even exceed human performance. Medical, industrial, forensic, security, as well as other applications seem to offer problems of this type. If the accuracy requirements are well defined, the it becomes easier for the short-listing process. Knowing the relative significance of false matches versus omitted true matches is particularly important. In the case of Cancer Identification through image analysis, the result is going to determine the life or death of a patient, where the effects of false positives or false negatives could be highly detrimental. If the computational requirements are well defined, this would also lead to efficient retrieval. In many cases instant response is not needed. For example, in a medical application it may take well over an hour to produce an image, so waiting another hour to find matches in a database is not particularly difficult. In security applications a fast response is often necessary but in that case the database is proprietary (not a public collection) and it may be organized accordingly for fast retrieval.

But this is not true in most cases, hence the need for a retrieval methodology that analyzes and determines the context of images is necessary.

As discussed by [5], for text-based retrieval string and standard query are easy, but because image database is hard to access with query language arbitrarily made by the user, you should enter the keywords that can describe the needed image. Besides, with incapability of expressing the features of image, such as color, texture and shape, consistently, it is being gradually converted in recent image retrieval system into content-based retrieval that uses the image content as features [4].

A study according to [24] points out that, modern computer vision research has its origins in the early 1960s. The earliest applications were pattern recognition systems for character recognition in office automation related tasks [9,17].Early work by Roberts in the 1960s [8] first identified the need to match two-dimensional features extracted from images with the three-dimensional representations of objects. Subsequent research established the practical difficulties in reliably and consistently accomplishing such a task, especially as the scene complexity increased, as the illumination variability increased, and as time, cost, and sensor noise constraints became more prevalent. 


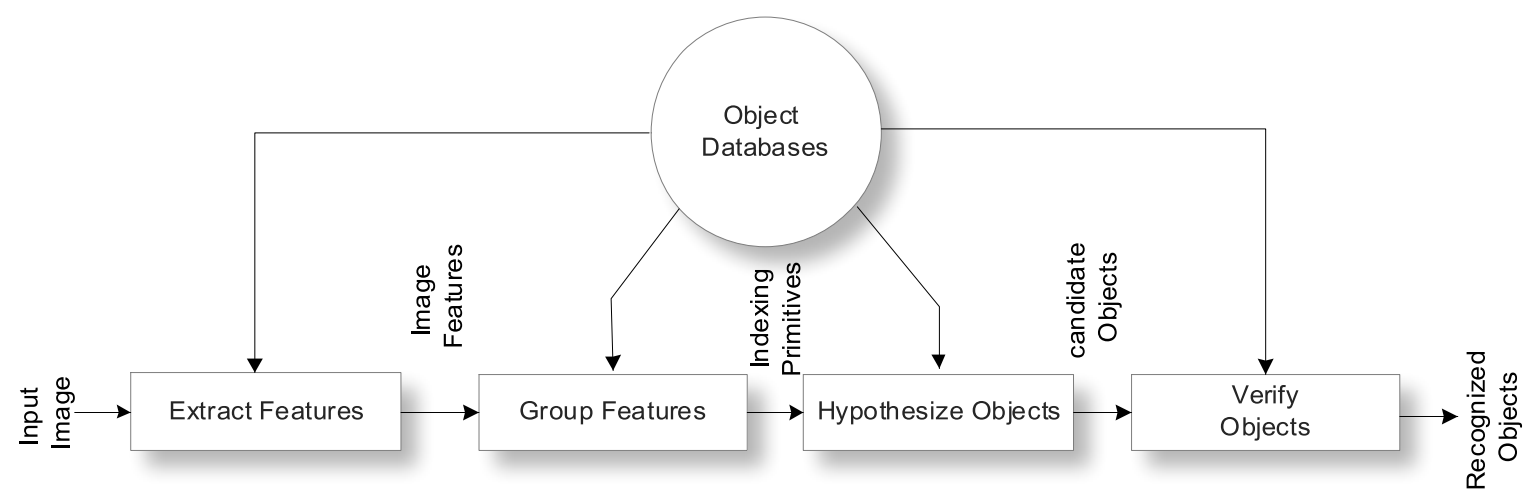

Figure 1: The components used in a typical object recognition system[21,22]

Early systematic work on vision systems is also traced to the Hitachi labs in Japan where the term machine vision originated, to distinguish its more pragmatic goal of constructing practical applications [18], as compared to the more general term computer vision, popularly used to also include less pragmatic goals. An early research thrust in 1964 involved the automation of the wire-bonding process of transistors, with the ultimate goal of replacing human workers. Even though the automated system achieved 95\% accuracy in lab tests, this was deemed too low to replace human workers. By 1973 however, fully automated assembly machines had been constructed [19], resulting in the world's first image-based machine for the automatic assembly of semiconductor devices.

Early recognition systems also appeared in biomedical research for the chromosome recognition task [20,21]. Even though this work initially had limited impact, its importance became clearer later. Recognition technologies are also successfully used in the food industry (e.g., for the automated classification of agricultural products [22]), the electronics and machinery industry (for automated assembly and industrial inspection purposes [23]), and the pharmaceutical industry (for the classification of tablets and capsules) [18].

According to [5], representative features of image in use include color distribution for image, object shape in image, general spatial layout, texture, etc. Since shape, spatial layout, texture, etc. are hard of calculation and complicated, method of using invariant color against translation, rotation and scale in the image is chiefly preferred.

Of all content-based image methods using color features, the most popularly used is the one that uses histogram. However, methods proposed in existing studies have a drawback of being sensitive to brightness of light and object size in the image, so approaches to supplement these are being proposed [25].

There are many kinds of clustering method at present time, which can be categorized into partitioning method, hierarchical method, density-based method, grid-based method, modelbased method, and subspace clustering method. Partitioning algorithms construct a partition of a database of $\mathrm{N}$ objects into a set of $\mathrm{K}$ clusters, they firstly make sure the number $\mathrm{K}$ of clusters. Hierarchical algorithms (BIRCH[26], CURE [27] ) are to decompose data set hierarchically. The disadvantage is that the termination condition has to be specified and it is ordersensitive. Density based algorithms (DBSCAN[28],
DENCLUE [29], CURD[30], CODU[31] ) rely on a densitybased notion of clusters, which control the cluster increasing according to the density threshold. Grid-based algorithms (STING[32], WaveCluster[33]) quantize the space into a finite number of cells and then do all operations on the quantized space. They depend only on the number of cells in each dimension in the quantized space.

For real high dimension data set, it is very difficult to specify the parameters of the number of clusters, agglomerative and divisive threshold, density threshold, and the grid granularity by prior knowledge. The common use of the Euclidean distance for computing a similarity measure appears unjustified. In high dimension, the more scattering data distribution is, the poorer the performance of clustering is. So, it is realistic to consider subspace clustering. CLIQUE[34] is a subspace clustering method that integrate density-based and grid-based methods. It is very effective for high dimension data in large database. CLIQUE extracts clustering units in every subspace from top to bottom. For extracting the clusters in ddimension, the clusters in all (d-1)-dimension must first be obtain, this is going to make the spatial and time efficiency become very low. Meanwhile, user must preset two parameters before clustering,namely, inter-cluster distance and density threshold. These parameters are sensitive to sample data distribution. And it is difficult for user to set these parameters. But CLIQUE method is insensitive for data order. ClusterTree is a new indexing approach representing clusters generated by any existing clustering approach. It supports effective and efficient image retrieval. Lots of clustering algorithms have been developed, and in most of them some parameters should be determined by hand.

[10] propose a new ClusterTree structure, which based on the improved CLIQUE and avoids any parameters defined by user. Using multi-resolution property of wavelet transforms, the proposed approach can cluster at different resolution and remain the relation between these clusters to construct hierarchical index. [10] integrates the characteristic of hierarchical, grid-based, and subspace-clustering methods, and there is not the parameters input by users. It also proposes to identify some smaller clusters.

[11] proposes the use of Correspondence Analysis (CA) for large scale content-based image retrieval. Correspondence Analysis is a useful method for analyzing textual data and we adapt it to images using the SIFT local descriptors. CA is used 
to reduce dimensions and to limit the number of images to be considered during the search step. An incremental algorithm for $\mathrm{CA}$ is proposed to deal with large databases giving exactly the same result as the standard algorithm. It also integrates the Contextual Dissimilarity Measure in the search scheme in order to improve response time and accuracy.

The words computed from images are called visual words and form a vocabulary of $\mathrm{N}$ words. This computation is made in two steps: (i) computation of local descriptors for a set of images and (ii) vector quantization of those descriptors into clusters which are called, by definition, visual words.

CA is applied on the documents and the visual words. Besides, CA provides some relevant indicators: every word or document represented as a point in the low dimensional factor space can be characterized by its contribution to the inertia of an axis or its quality of representation (Greenacre, 2007; Morin, 2004). [11] uses the incremental CA for performing this analysis.

[12] presents a mechanism that deals with imprecise information in a content based image retrieval system. The imperfection in images is related to the very representation of images, as well as to the user's preferences expressed directly with the use of natural language elements or, what is more practical, indirectly in the features of an image composed using the query-by-example paradigm.

Images are assumed to be represented by collections of objects extracted from them. These objects are characterized by some low-level visual features and they are assigned to semantically meaningful classes by an automatic procedure. These objects are further characterized by their spatial relationships, which play an important role in determining the matching between the images.

[13] combines the use of low-level descriptors and semantic metadata for similarity search. The architecture has three levels: content-based, semantic data and an interface integrating them. Several database User Defined Types (UDT) and operations are defined for that purpose. It uses an object relational database for data storage.

[14] proposes to exploit an approach to perform approximate similarity search in metric spaces developed by $[35,36]$. The idea at the basis of these techniques is that when two objects are very close one to each other they 'see' the world around them in the same way.

Accordingly, it uses a measure of dissimilarity between the views of the world at different objects, in place of the distance function of the underlying metric space. To employ this idea the low level image features (such as colors and textures) are converted into a textual form and are indexed into the inverted index by means of the Lucene search engine library. The conversion of the features in textual form allows to employ the Lucene's off-the-shelf indexing and searching abilities with a little implementation effort. In this way, it provides a robust information retrieval system that combines full-text search with contentbased image retrieval capabilities. [14] can be considered an hybrid approach, even if it is mainly based on a space transformation it also adopts techniques to reduces the amount of data accessed.

[15] propose a term selection model to help select terms in the documents that describe the images to improve the contentbased image retrieval performance. A general feature selection model is presented, after which, a method for training document collections is put forward, followed by selecting and ranking the terms using the Kullback-Leibler Divergence.
ImageCLEF is a continuing image retrieval track, run as part of the Cross Language Evaluation Forum (CLEF) campaign. This track evaluates retrieval of images described by text captions based on queries in a language. The goal of its retrieval track is to explore and study the relationship between images and their captions during the retrieval process.

In [16], a novel content based image retrieval (CBIR) system based on a new Multiscale Geometric Analysis (MGA)-tool, called Ripplet Transform Type-I (RT) is presented. It satisfies the multiresolution, good localization, high directionality, general scaling and support, anisotropy and fast coefficient decay properties. This makes RT as an effective tool to represent images or $2 \mathrm{D}$ signals, and motivated us to use it, to represent the images in the DB. To improve the retrieval result, a fuzzy relevance feedback mechanism (F-RFM) is also implemented. Fuzzy entropy based feature evaluation mechanism is used for automatic computation of revised feature's importance and similarity distance at the end of each iteration.

\section{DIRECTIONS FOR FUTURE RESEARCH}

In the area of image analysis there is another important characteristic of successful applications. They tend to be in situations where human recognition relies on the meticulous study of details rather than a general overview of the scenario. Thus image analysis has found successful applications in industrial inspection and biometrics, especially fingerprint recognition. Automatic fingerprint identification (retrieving a match to a fingerprint from a database) is used in practice by the FBI and there are several commercial products for fingerprint verification. In contrast to text retrieval that relies on linguistic expressions of human concepts, there is no well defined set of rules to formalize the content of images.

For any automatic image processing we need a mapping between human impressions ("semantics") and quantities that can be computed ("features"). Successful pattern recognition relies on such quantities. Statistical techniques may be used for fine tuning the parameters necessary to design such features, but they cannot uncover the structure of the mapping. In generating such features we must also specify the classes of images we are dealing and this, in turn, facilitates the proper selection of "learning" samples.

For bridging the semantic gap we can rely on Ontology, Semantics and association rules to identify the context between the objects rather than treating the objects individually. Using Semantics helps us to avoid the inherent inconsistencies in the data that is being used. Ontology provides a domain specific knowledge thereby helping us to concentrate on the details rather than having just an overview of the scenario. We can extract all the objects in an image and apply association rules to identify the context and then use our segmentation and tagging approach to tag the images appropriately.

For defining the accuracy requirements and for careful scrutiny of the images we need active interaction with the user through a relevance feedback mechanism. Inconsistency resolution is a process in which users must be given a prominent voice. Depending on their individual preferences (which are subject to individual scenarios), users must be allowed to decide how inconsistencies should be resolved. Empirical Bayesian Learning for Fuzzy based relevance feedback could be used. [42] proposes an image retrieval technique that considers the domain name system space adjustment technique. 
Further, usage of Association rule mining techniques [41] can be incorporated for analyzing and determining the dependencies between objects in the images and between images. Segmentation and tagging can be used for providing labels to images and objects in images, hence will help in future analysis. Employing Bayesian learning [38, 39] for fuzzy based relevance feedback can be used for obtaining a higher degree of accuracy.

\section{IMPLEMENTATION SUGGESTIONS \\ 5.1. Cluster or Cloud based Implementation}

A Cluster based or Cloud based implementation could be studied because of the highly distributed and large scale nature of the image databases and the complexity involved in the process of CBIR. This provides excellent Scalability, Fault Tolerance, and Flexibility and also improves performance to a greater extent and thereby makes it possible for real time usage.

\subsection{Swarm Intelligence}

Swarm Intelligence [39] based on ANT Colony Optimization (ACO)[40] could be used for Distributed CBIR. For Load Balancing, we can employ a Alienated Ant Colony Optimization (AACO) where the distribution is found to be optimal. Usually ACO works based on the principle that ants will leave a pheromone particle that alerts other ants about the path availability. Higher the concentration of the pheromone, greater the number of ants taking that path.

But this is not suitable for load balancing applications and hence we propose a reverse approach to be used. Our approach is an inverse of the ACO called AACO (Alienated ACO) where the ants or agents will move in paths less taken so that distributed retrieval with load balancing could be achieved.

\subsection{Load Balancing}

A Distributed, Dynamic, Global and Highly Scalable Load Balancing algorithm will make CBIR much more versatile and usable in the real time scenarios. Issues include the heterogeneity of the cluster or grid and the Workload Distribution among the nodes and the implementation of a dynamic updating scheme for active workload distribution based on current workloads of the nodes. This could be achieved using Swarm Intelligence or Swarm Optimization Techniques.

\section{CONCLUSION}

CBIR is an emerging trend that is about to occupy one of the main slots in the search process. This paper discusses the various problems faced by a CBIR system. It also discusses the various methods that have been proposed to provide an efficient CBIR system. Their merits, demerits and mode of working have been discussed. Directions for future research have been put forth and best implementation mechanisms that can be used for providing an efficient CBIR system has been discussed.

\section{REFERENCES}

[1] Jain and A.Vailaya, Aug. 1996, "Image Retrieval Using Color and Shape,"Pattern Recognit., Vol. 29, No. 8, pp. 1233-1244.

[2] I.J. Cox, et al., 2000, "The Bayesian image retrieval system, PicHunter: theory, implementation, and psychophysical experiments." IEEE, Trans. On Image Processing. 9, p. 20-37.
[3] Weber R, Schek J-J, Blott S, August 1998, “Aquantitative analysis and performance study for similaritysearch methods in high-dimensional space." In: Proc int'l conf very large databases, New York, 24-27, pp 194-205.

[4] Kaplan LM, Murenzi R, Namuduri KR, 1997, "Fast texture dadabase retrieval using extended fractal features." Proc SPIE Conf On Electric Imaging: Storage \& Retrieval for Image and Video Database IV 3312:162-173, San Jose, CA.

[5] Yang-Hoon Kim \& Hyuk-Jun Kwon \& Jong-Gu Kang \& Hangbae Chang, "The study on content based multimedia data retrieval System," Multimed Tools Appl 57:393-405, DOI 10.1007/s11042-011-0758-5,2012.

[6] J. Tsotsos, John Wiley and Sons, 1992, "The Encyclopedia of Artificial Intelligence," pp. 641663, Chapter: Image Understanding.

[7] S. Dickinson,1999,"What is Cognitive Science?", Basil Blackwell Publishers, pp. 172-207, Chapter: Object Representation and Recognition.

[8] L.G. Roberts, Machine Perception of Three Dimensional Solids, Ph.D. thesis, 1963, "Massachusetts Institute of Technology".

[9] L.G. Roberts, 1960,"Pattern recognition with an adaptive network," in: Proc. IRE International Convention Record, pp. 66-70.

[10] Xu, De Xu, and Enai Lin , A. Gagalowicz and W. Philips (Eds.), Hongli, 2007, “An Applicable Hierarchical Clustering Algorithm for Content-Based Image Retrieval,": MIRAGE 2007, LNCS 4418, pp. 82-92. (C) Springer-Verlag Berlin Heidelberg 2007

[11] Nguyen-Khang Pham, Annie Morin, Patrick Gros, and Quyet-Thang Le, F. Guillet et al. (Eds.), "Intensive Use of Correspondence Analysis for Large Scale Content-Based Image Retrieval, Advances in Knowledge Discovery and Management," SCI 292, pp. 57-76.

[12] Tatiana Jaworska1, Janusz Kacprzyk1, Nicolas Mar'in2, and S_lawomir, Zadro'zny, E. H"ullermeier, R. Kruse, and F. Hoffmann (Eds.), 2010, "On Dealing with Imprecise Information in a Content Based Image Retrieval System", IPMU 2010, LNAI 6178, pp. 149-158. Springer-Verlag Berlin Heidelberg 2010

[13] Carlos E. Alvez1 and Aldo R.Vecchietti2, R. Setchi et al. (Eds.), 2010, "Combining Semantic and Content Based Image Retrieval in ORDBMS,": KES 2010, Part II, LNAI 6277, pp. 44-53.

[14] Gennaro, Giuseppe Amato, Paolo Bolettieri, and Pasquale Savino, M. Lalmas et al. (Eds.), Claudio 2010,"An Approach to Content-Based Image Retrieval Based on the Lucene Search Engine Library", ECDL, LNCS 6273, pp. 55-66, 2010.

[15] Qinmin Vivian Hu1, Zheng Ye1,2, and Xiangji Jimmy Huang1, A. An et al. (Eds.),2010, "Enhancing ContentBased Image Retrieval Using Machine Learning Techniques,” AMT 2010, LNCS 6335, pp. 383-394.

[16] Chowdhury, Sudeb Das, and Malay Kumar Kundu, M.K. Kundu et al,2012, "Interactive Content Based Image Retrieval Using Ripplet Transform and Fuzzy Relevance Feedback, Manish," Eds.: PerMIn 2012, LNCS 7143, pp. 243-251. 
[17] J.T. Tippett, D.A. Borkowitz, L.C. Clapp, C.J. Koester, A.J. Vanderburgh (Eds.,1965, "Optical and ElectroOptical Information Processing," MIT Press

[18] M. Ejiri,2007,"Machine vision in early days: Japan's pioneering contributions", in: Proc. 8th Asian Conference on Computer Vision (ACCV).

[19] S. Kashioka, M. Ejiri, Y. Sakamoto,1976,“A transistor wire-bonding system utilizing multiple local pattern matching techniques," IEEE Transactions on Systems, Man and Cybernetics 6 (8),562-570.

[20] G. Gallus, "Contour analysis in pattern recognition for human chromosome classification," Appl Biomed Calcolo Electronico 21968.

[21] G. Gallus, G. Regoliosi, "A decisional model of recognition applied to the chromosome boundaries," Journal of Histochemistry \& Cytochemistry 221974.

[22] A. Jimenez, R. Ceres, J. Pons,2000, "A survey of computer vision methods for locating fruits on trees," IEEE Transactions of the ASABE 43 (6), ) 1911-1920.

[23] E.N. Malamas, E.G.M. Petrakis, M. Zervakis, L. Petit, JD. Legat,2003,"A survey on industrial vision systems, applications and tools," Image and Vision Computing 21 (2) $171-188$

[24] 50 Years of object recognition: Directions forward $q$, Alexander Andreopoulos a, $\Uparrow$, John K. Tsotsos b,2013, “Computer Vision and Image Understanding”, 117 827-891,http://dx.doi.org/10.1016/j.cviu.2013.04.005

[25] Wang Z, Jia K, Liu P,2009,“An effective web contentbased image retrieval algorithm by using SIFT feature." Softw Eng 1:291-295.

[26] Tian Zhang, Raghu Ramakrishnan, and Miron Livny. BIRCH,1996, “An Efficient Data Clustering Method for Very Large Databases." In Proceedings of the 1996 ACM SIGMOD International Conference on Management of Data, pages 103-114, Montreal, Canada.

[27] Guha S, Rastogi R, Shim K. CURE ,1998,“ an efficient clustering algorithm for large databases." Proc. of the ACM SIGMOD Int'l Conf. on Management of Data. Seattle: ACM Press, 73 84.

[28] Ester M, Kriegel H, Sander J, Xu XW.1996,“A densitybased algorithm for discovering clusters in large spatial databases with noise." Proc. of the 2nd Int'l Conf. on Knowledge Discovery and Data Mining (KDD'96). Portland: AAAI Press, 226 231.

[29] Hinneburg A, Keim D.1998,"An efficient approach to clustering in large multimedia databases with noise." Proc. of the 4th Int'l Conf. on Knowledge Discovery and Data Mining (KDD’98). New York: AAAI Press, 58 65.

[30] Ma S, Wang TJ, Tang SW, Yang DQ, Gao J.,2003, “A fast clustering algorithm based on reference and density. Journal of Software,"14(6):1089 1095. in china.
[31] Liu Kan, Zhou Xiao Zheng , and Zhou DongRu., Oct1 2003,"Clustering by Ordering Density-Based Subspaces and Visualization Journal of Computer Research and Development,"Vol. 40, No. 10, 1509 1513, in china.

[32] Wang W, Yang J, Muntz RR. STING:,1997,“A statistical information grid approach to spatial data mining." Proc. of the 23rd Int'l Conf. on Very Large Data Bases. Athens: Morgan Kaufmann,186 195.

[33] Sheikholeslami G, Chatterjee S, Zhang AD WaveCluster:,1998,“Amulti-resolution clustering approach for very large spatial databases." Proc. of the 24th Int'l Conf. on Very Large Data Bases. New York: Morgan Kaufmann, 428 439.

[34] Rakesh A, Johanners G, Dimitrios G, Prabhakar R.1998,"Automatic subspace clustering of high dimensional data for data mining applications." Proc. of the 1998 ACM SIGMOD Int'l Conf. on Management of Data. Minneapolis: ACM Press. 94 105.

[35] Amato, G., Savino, P.2008:"Approximate similarity search in metric spaces using inverted files." In: Proceedings of the 3rd International Conference on Scalable Information Systems (InfoScale 2008), pp. 1-10. ICST

[36] Chavez, E., Figueroa, K., Navarro, G,2007: "Effective proximity retrieval by ordering permutations." IEEE Transactions on Pattern Analysis and Machine Intelligence 30, 1647-1658.

[37] Zhen Jis and Arjjuna Balasuriya,2004,"Bayesian Learning for Object Based Image ." Proceedings of the 2004 IEEE, Conference on Cybernetics and Intelligent Systems Singapore, 1-3 December.

[38] Abhishek Singh, Padmini Jaikumar and Suman K Mitra, "A Bayesian Learning Based Approach for Clustering of Satellite ImagesSixth Indian Conference on Computer Vision," Graphics \& Image Processing, 978-0-7695-34763/08 \$25.00 (C) 2008 IEEE, DOI 10.1109/ICVGIP.2008.60

[39] Mr. Pankaj K. Bharne, Mr. V. S. Gulhane, Miss. Shweta K. Yewale, "Data Clustering Algorithms B ased On Swarm Intelligence," 978-1-4244-8679-3/11/\$26.00 (C)2011 IEEE

[40] Marco Dorigo and Thomas St"utzle ,"Book Review: Ant Colony Optimization," Genetic Programming and Evolvable Machines,Published by: MIT Press, 2004, ISBN 0-262-04219-3, 328 pages, 6, 459-460, 2005

[41] Muhammad Shaheen, Muhammad Shahbaz, Aziz Guergachi, "Context based positive and negative spatiotemporal association rule mining," Knowledge-Based Systems 37 (2013) 261-273

[42] N.Karthikeyan, Dr.R.Dhanapal,2013, "Image Retrieval of Domain Name System Space Adjustment Technique". International Journal of Computing, Vol. 5, No.3, pp.34 38 